\title{
conf-910730--24
}

\section{A SHTUTTER DESIGN FOR TIME DOMAIN STUDIES USING SYNCHROTRON RADIATION AT THE ADVANCED PHOTON SOURCE*}

ANL/CP- -72872

DE92 003405

J. R. Norris ${ }^{1}$, M. K. Bowman ${ }^{1}$, L. Chen ${ }^{1}$, J. Tang ${ }^{1}$, M. C. Thurnauer ${ }^{1}$, G. S. Knapp ${ }^{2}$, and P. A. Montano ${ }^{2}$ ${ }^{1}$ Chemistry Division

2Materials Science Division

Argonne National Laboratory Argonne, IL 60439

The submitted manuscript has been authored
by a contractor of the U. S. Govcrnment
under contract No. W-31-109-ENG-38.
Accordingly, the U.S. Government retains a
nonexclusive, royalty-free license to publish
or reproduce the published form of this
contribution, or allow others to do so, for
U.S. Government purposes.

SEPTEMBER 1991

trlp

Distribution:

1-3. D. R. Hamrin

4. B. D. Dunlap

5. M. B. Brodsky

6. P. A. Montano

7-13. Authors

14. Editorial Office

15. F. Y. Fradin

Submitted to 4th International Conference on Synchrotron Radiation Instrumentation (SRI), Chester, UK, July 15-19, 1991.

*Work Supported by the U.S. Department of Energy, BES-Materials Sciences, under Contract W-31-109-ENG-38.

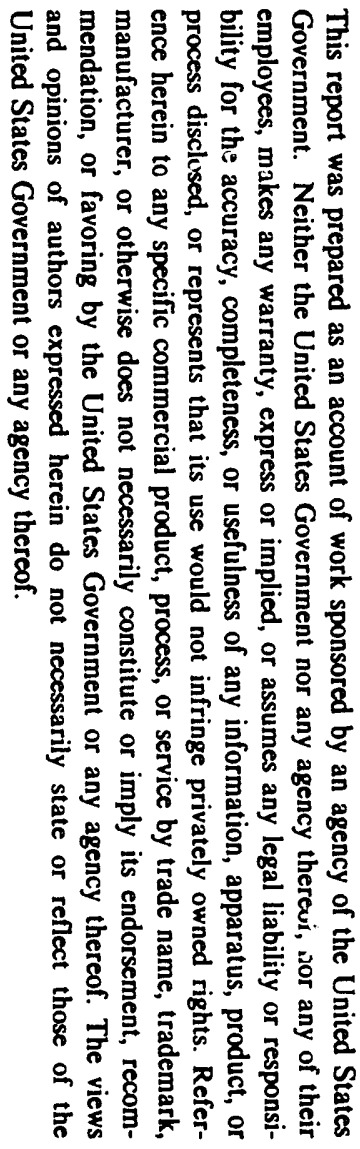




\section{A SHUTTER DESIGN FOR TIME DOMAIN STUDIES USING SYNCHROTRON RADIATION AT THE ADVANCED PHOTON SOURCE*}

J. R. Norris ${ }^{1}$, M. K. Bowman ${ }^{1}$, L. Chen ${ }^{1}$, J. Tang ${ }^{1}$, M. C. Thurnauer ${ }^{1}$, G. S. Knapp ${ }^{2}$, and P. A. Montano 2

${ }^{1}$ Chemistry Division

2Materials Science Division

Argonne National Laboratory

Argonne, IL 60439

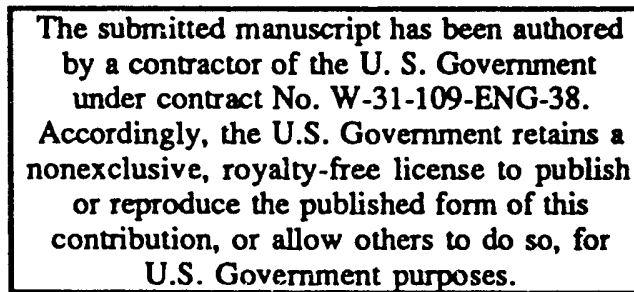

SEPTEMBER 1991

Submitted to 4th International Conference on Synchrotron Radiation Instrumentation (SRI), Chester, UK, July 15-19, 1991.

*Work Supported by the U.S. Department of Energy, BES-Materials Sciences, under Contract W-31-109-ENG-38. 
A SHUTTER DESIGN FOR TIME DOMAIN STUDIES USING SYNCHROTRON RADIATION AT THE ADVANCED PHOTON SOURCE

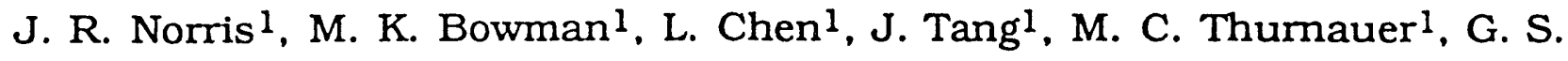
Knapp ${ }^{2}$, and P. A. Montano 2

1) Chemistry Division and 2) Materials Science Division, Argonne National Laboratory, Argonne, IL 60439, USA.

Abstract.

In general a variable repetition rate of the $x$-ray bunches is needed to explore time domain problems using $x$-ray radiation. In some instances the results of several hundreds or thousands of $x$-ray pulses must be averaged requiring the sample to be in the same time dependent state each time the monitoring pulse strikes. In the most advanced and most detaile 1 version of this type experiment an intense laser pulse would be used to create an excited state from a relaxed ground state. An additional "probe-pulse" that follows the laser "pump-pulse" would examine the sample. The important point is that before a second $x$-ray pulse hits the sample, the system must return to its initial relaxed ground state prior to another laser pulse in order to prepare the same excited state again. Otherwise the second $x$-ray probes a different condition of the system than the previous $x$-ray bunch such that any data averaging scheme would be invalid. Our system is primarily designed for the Advanced Photon source (APS)at Argonne Natinnal Laboratory. In the 20-bunch mode of APS an x-ray pulse will occur every 177 nanoseconds, requiring each edge of a conventional two-blade "shutter" to travel at least $0.25 \mathrm{~mm}$ in $\sim 177$ nanoseconds. Our key design principle employs a subsonic, rotating mirror whose period is slaved to the synchrotron intra-pulse period. The synchrotron $x$-ray bunches will be reflected a distance of about two meters to a narrow $0.5 \mathrm{~mm}$ slit just in tront of the sample. The time that the reflected synchrotron spends striking the slit is given by $1 /(4 \pi r f)$ where $r$ is the radius from the center of the spindle to the slit, and $f$ is the frequency of rotation of the spindle. A mirror rotating at a low $7,500 \mathrm{rpm}(125 \mathrm{~Hz})$ is sufficient to select a single synchrotron pulse. The very precise phase stability required by this method is currently available in rotating mirror devices for laser scanning. 
Introduction

A most striking aspect of current synchrotron research is the relatively small number of time domain studies based on the pulsed nature of the $\mathrm{x}$-ray radiation. Synchrotron radiation has been used to perform timeresolved experiments employing position sensitive detectors 1.2 with the best time resolution (attained with a CCD detector) of the order of $25 \mu \mathrm{sec}^{3}$. Time-resolved $x$-ray measurements have been carried out on semiconductor crystals during laser annealing. Larson et $\mathrm{al}^{4,5}$ used the pulsed time structure of CHESS to study laser annealing in Si and Ge crystals. A single bunch operational mode was employed to perform such studies, with a time resolution of about 250 ns. Recently, Kawado et $a^{6}$ used a plastic scintillator detector with a rapid decay time (of the order of nanoseconds) to investigate lattice deformation of a semiconductor crystal under laser irradiation, with a time resolution of about 50ns. Laue diffraction patterns with good signal to noise from a single pulse using undulator radiation are possible. 7 This problem has been treated in some detail in the literature, primarily by $\mathrm{J} . \mathrm{K}$. Moffat and J. Helliwell ${ }^{8}$ and D. M. Mills, A. Lewis, A. Harootunian, J. Huang and $B$. Smith 9 . If such single crystal studies are successful little questior. exists about their importance. Presently, no x-ray based structural studies have been successful on such a short time scale. At considerably slower time scales a few preliminary results on single crystals have been encouraging as recently reviewed in the literature 8,9 .

Although synchrotrons represent an unrivaled source of intense, pulsed $x$-rays, full potential of this valuable tool has not been realized in time deperident applications. Whereas other synchrotrons have pulse widths of the order of 100 picoseconds or longer, at the APS of ANL the pulse width of the radiation bunches is to be less than 60 picoseconds; as a resilt, the ultimate time resolution of the APS synchrotron also will be less than 60 picoseconds. Realization of such an ultimate time resolution requires that the sample be irradiated by $x$-rays only once per chemical or physical event whose time sequence is to be probed. In order to accomplish this class of experiments an x-ray "shutter" for synchrotrons has been designed by us to single out a particular radiation bunch among the millions contained in the continuous train of synchrotron $x$-ray bursts. The ability to control a single 
bunch of $\mathbf{x}$-rays provides either a monitoring "probe-pulse" or an excitation "pump-pulse" for investigating dynamics in chemical, physical and biological systems with sub-nanosecond time resolution. Cur "shutter" will permit the exploration of the folluwing three major areas: 1) time domain structure determination using spectroscopy (e.g., EXAFS and XANES); 2) timeresolved crystallography; 3 ) $\mathrm{x}$-ray radiation damage studies.

Pulsed lasers with widths ranging from 15 nanoseconds to less than 100 femtoseconds are routinely employed in time domain studies to examine chemistry, to initiate chemistry or both. Many unpredicted experimental breakthroughs have resulted from such time domain investigations using pulsed lasers, including the unraveling of the primary events of photosynthesis and vision. In contrast to pulsed lasers and despite the pulsed nature of synchrotrons, little or no chemistry has been explored taking full advantage of the short pulse width of synchrotron radiation. The repetition rate for synchrotron radiation bunches is approximately 300,000 times per second or faster. In other words, the minimum time between synchrotron bunches is about 3.5 microseconds or shorter. For conventional synchrotron investigations this rapid bunch rate is an advantage since the high repetition rate leads to a correspondingly high average $x$-ray photon flux. Actually, most major synchrotron facilities operate in a multi-bunch mode such that this 3.5 microseconds is reduced proportional to the number of bunches loaded into the synchrotron ring. For example the APS will primarily operate in a 20 bunch mode such that the time interval between bunches is 20 times shorter, 1.e., about 177 nanoseconds. The shorter this inter-bunch interval, the more difficult is the problem of exploring the time domain because the more difficult becomes the task of selecting a single radiation bunch.

In complete contrast, repetition rates of many pulsed lasers used in time domain optical spectroscopy range from 10 to 1000 per second. The necessity of such low repetition rates is dictated by the time required for the chemical or physical process of the sample to recover to the original state or condition. Typically a single pulse of radiation is not sufficient to provide enough signal-to-noise to complete an experiment. Instead, the experimental results of several hundreds or thousands of pulses must be 
averaged. The fundamental requirement of this type averaging experiment is that the sample must be in the same state each time the monitoring pulse strikes the object of study. The most common means for fulfilling the requirement of exactly the same sample condition prior to each examining pulse is to investigate systems with reversible chemistry or physics. For example, a laser "pump-pulse" could be used to create an excited state in a sample that initially resides in a relaxed ground state. In such an example the excited state is the condition of the sample of interest; thus one needs an additional "probe-pulse" to follow immediately the laser "pumppulse"excitation that has prepared the sample in the desired excited state. For instance, after the laser "pump-pulse" strikes the sample a synchrotron "probe-pulse" could examine the sample as depicted in Figure 1. For example, the $\mathrm{x}$-ray "probe-pulse" may be part of a time domain EXAFS experiment. However, before a second $x$-ray pulse can strike the sample, the system under study must return to the initial relaxed ground state such that another laser "pump-pulse" may strike the sample and prepare the same "excited" state and at the same point in time after its "birth". Otherwise the second $x$-ray "probe-pulse" would measure a different state or a different point in time of the system than the previous "probe-pulse" and thus the data averaging process would become invalid. If a single pulse is adequate, the device we are proposing will just as easily provide one pulse to the sample.

The problem facing the experimentalist is that typically longer than 3.5 microseconds is required for systems (including all side reactions) to relax completely to the initial state. Photosynthesis is a good example. Although a light pulse can induce an electron transfer reaction in 2.8 picoseconds, relaxation back to the initial step requires approximately 15 nanoseconds for the main reaction of the singlet manifold and about 100 microseconds for the accompanying secondary photochemistry of the triplet manifold. This example and the approximate times are valid only for specially conditioned reaction centers. Even though only a small proportion of the total chemistry occurs in the triplet manifold, light repetition at intervals of less than about 100 microseconds would result in a significant fraction of the molecules residing in a steady state dominated by the longest lived species, i.e., the triplet state in this instance. Since synchrotron 
facilities do not provide the experimentalist with the means to control the time interval between radiation bunches, only a few chemical or physical systems, i.e., those with very fast recovery times and those with no additional reactivity channels, can be explored. Unfortunately, systems most interesting in chemistry have relaxation rates considerably longer than 3.5 microseconds. Also one must keep in mind that the standard method of operation is with the synchrotron ring loaded in a multi-bunch mode such that the inter-pulse interval is considerably shorter than 3.5 microseconds. Consequently, the relaxation and recovery rate of the chemistry must be even faster. In addition, a host of other technical problems arise, such as the unavailability of high power lasers that can generate a laser pulse every 3.5 microseconds or faster as required by the data averaging technique. Thus, this inability to control the repetition rate of the synchrotron radiation has completely excluded considerable chemistry and physics from being explored at synchrotron facilities.

To overcome this stumbling block, we have thus developed a general device which will appropriately "shutter" synchrotron radiation of energies up to $20 \mathrm{Kev}$ and thereby provide a single bunch of synchrotron radiation on demand. In the study of reversible processes, the device must select a single bunch of x-ray at some pre-determined frequency, for example 10 times a second. Moreover, we have chosen to work in the multiple bunch mode of the synchrotron operation in order to insure that sufficient experimental time can be devoted to this new class of synchrotron research. We note that the APS design is quite favorable for the multiple bunch mode since the interval between multiple bunches is a minimum of 177 nanoseconds. Another advantage for our 'single' bunch operation results from the intense nature of the APS bunches. Finally we mention that working with the multiple bunch mode operation has increased the design constraints on our x-ray "shutter".

\section{Shutter Design}

These technical details are for the APS at Argonne National Laboratory. The dimensional size of the light beam to be chopped is taken to be $0.5 \mathrm{~mm}$. Such a narrow beam results from use of an undulator designed for APS. Also 
since we assume the 20 bunch mode, a light pulse will occur every 177 nanoseconds, requiring each edge of a two-blade "shutter" to move at least $0.25 \mathrm{~mm}$ in 177 nanoseconds. In other words, the blades would have to travel approximately four times faster than the speed of sound. Even though rotational speeds up to $1,200,000 \mathrm{rpm}(20,000 \mathrm{~Hz})$ are possible, normally these devices do not exceed the speed of sound in air (or a gas such as helium). Thus, we reject this brute force approach. Instead, we have developed a "shutter" design that circumvents the problem of using mechanical devices with speeds greater than that of sound. Figure 2, illustrating our key design principle, features a rotating, four/sided, mirrored spindle. The rotational period of the mirrored spindle is slaved to the synchrotron intra-pulse period. The synchrotron-light pulses will be reflected a distance of two meters to a narrow $0.5 \mathrm{~mm}$ slit behind which we will place our sample. The speed of the reflected synchrotron pulse traveling across the slit is given by $1 /(4 \pi \mathrm{r} f)$ where $r$ is the radius from the center of the spindle to the slit, and $f$ is the frequency of rotation of the spindle. A small spindle rotating at a low $7,500 \mathrm{rpm}(125 \mathrm{~Hz})$ is sufficient to select a single synchrotron pulse. The reflected pulse at the slit moves from position 1 to position 2 eight times faster than the speed of sound even though the rotating spindle travels significantly slower than sound. This technique solves the speed requirement for the "shutter".

However, our approach introduces a new technical problem, spatial stability. The reflected radiation bunch must strike the $0.5 \mathrm{~mm}$ slit within an accuracy about $0.05 \mathrm{~mm}$. Since the total circumference is $2 \pi \mathrm{r}$, an accuracy of 1 part in 125,000 is required for a radius of two meters. Such high stability has been achieved in a commercial computer controlled optical mirror rotator. All that need to be done is to equip the commercially available system with an $\mathrm{x}$-ray mirror and prepare a pre-shutter, i.e., a slow shutter, to absorb the bulk of the $\mathrm{x}$-ray radiation damage and heat. Since $\mathrm{x}$ ray mirror technology exists for energies up to approximately $20 \mathrm{kev}$, many new and novel uses of synchrotrons will be permitted by our proposed synchrotron "shutter". Examples are time domain $\mathrm{x}$-ray diffraction and scattering as well as picosecond laser spectroscopy of the effects of synchrotron radiation on a variety of chemical substances. One of our 
principal goals is the determination of chemical structures by high resolution $\mathrm{x}$-ray diffraction during chemical reactions.

\section{Conclusions}

These pump-probe experiments, with a combination of a synchrotron source and another source such as light, heat, electric field, or pressure, can be used to study dynamics on the time scale of synchrotron pulse width. In various pump-probe experiments, a synchrotron pulse can be used as a pump to initiate a chemical reaction, or used as a probe to follow the change in 1 the system as a function of a relative time delay between the pump source, which creates change in the system, and the probe, the synchrotron pulse. We note that the time resolution of the experiments conducted in this pump-probe fashion is not limited by data collection time and detector response time. In this paper the examples we have presented arbitrarily emphasize laser light as the pulse accompanying the synchrotron pulse.

Clearly, several different techniques for time domain $\mathrm{x}$-rays investigations are possible ${ }^{10}$. The previously briefly mentioned time dependant EXAFS experiment can be performed where the ultimate time resolution should be the synchrotron pulse width $(<60$ picoseconds at APS) and not the 1 to 0.001 second time resolution currently achieved in present cxperimental setups ${ }^{11}$. Again, the underlying means to accomplish all these time domain $\mathrm{x}$-ray experiments is the ability to repeat precisely the same experiment over and over while averaging all the time to enhance signal-tonoise. Similarly, the requirement on the synchrotron pulse repetition rate is the same as previour!y described. These alternative experiments are technically easier than those based on diffraction from single crystals and appear certain to will lead to successful results if the appropriate synchrotron "shutter" is developed. For these reasons primarily, we have devoted considerable effort in designing a suitable $x$-ray synchrotron "shutter" since we feel strongly that such a device is the key technical requirement for the development of a new branch of synchrotron experiments. 
Acknowledgements.

The authors acknowledge helpful discussions with D. Mills. This work was supported by the U.S. Department of Energy, Office of Basic Energy Sciences under contract W-31-109-ENG-38.

\section{References}

1. P. Hernandez, L. Lamelas, R. Clarke, P. Dimon, E. B. Sirota and S. K. Sinha, Phys. Rev. Lett. 58, 1220 (1987)

2. R. Clarke and F. Lamelas, to be published

3. B. Rodrici , C. Brizard, R. Clarke, and W. Lowe to be published.

4. B. C. Larson, C. W. White, T. S. Noggle, and D. M. Mills, Phys. Rev. Lett. 48, 337 (1982) .

5. J. Z. Tischler, B. C. Larson, and D. M. Mills, Appl. Phys. Lett. 52, 1785 (1988) .

6. S. Kawado, S. Kojima, T. Ishikawa , T. Takahashi, and S. Kikuta, Rev. Sci. Instrum. 60,2342 (1989).

7. G. S. Knapp and M. A. Beno in preparation.

8. J. K. Moffat and J. Helliwell, Topics in Current Chemistry, 151, pp 6174, (1989)

9. D. M. Mills, A. Lewis, A. Harootunian, J. Huang and B. Smith, Science 1984, pp 811-13

10. D. M. E. Szebenyi, D. Bilderback, A. LeGrand, K. Moffat, W. Schildkamp and T.-Y. Teng, Trans. Am. Cryst. Ass. 24 167-72, (1989).

11. J. McBreen, W. E. O'Grady, G. Tourillon, E. Dartyge, A. Fonntaine, and K. I. Pandya, J. Phys. Chem , 93, 6308-11, (1989). 
Figure Captions

Figure 1. APS timing system (not drawn to scale).

Figure 2. Synchrotron Shutter / Mirror rotated such that in position 1 a pulse is selected and in position 2 a pulse is rejected. 


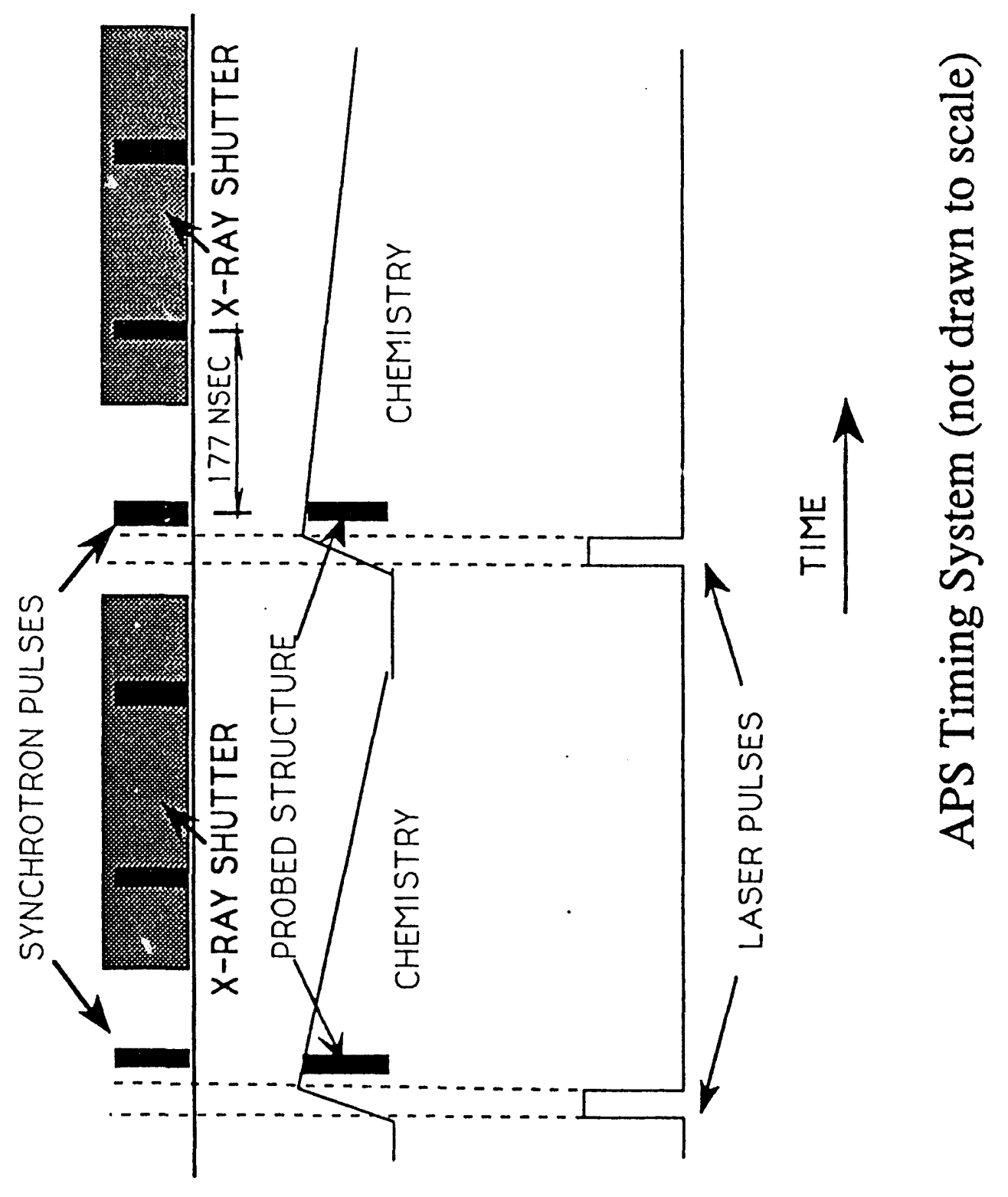




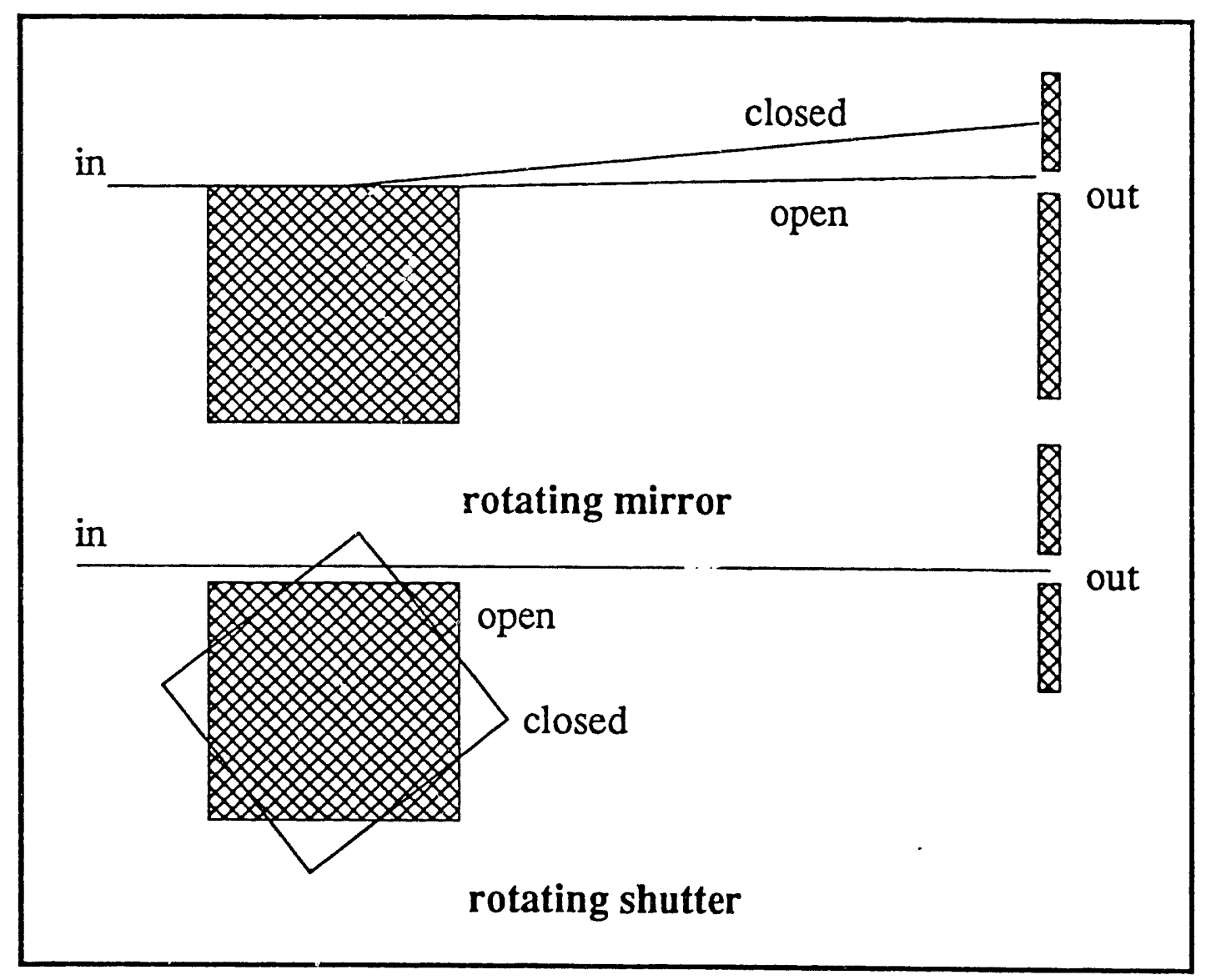



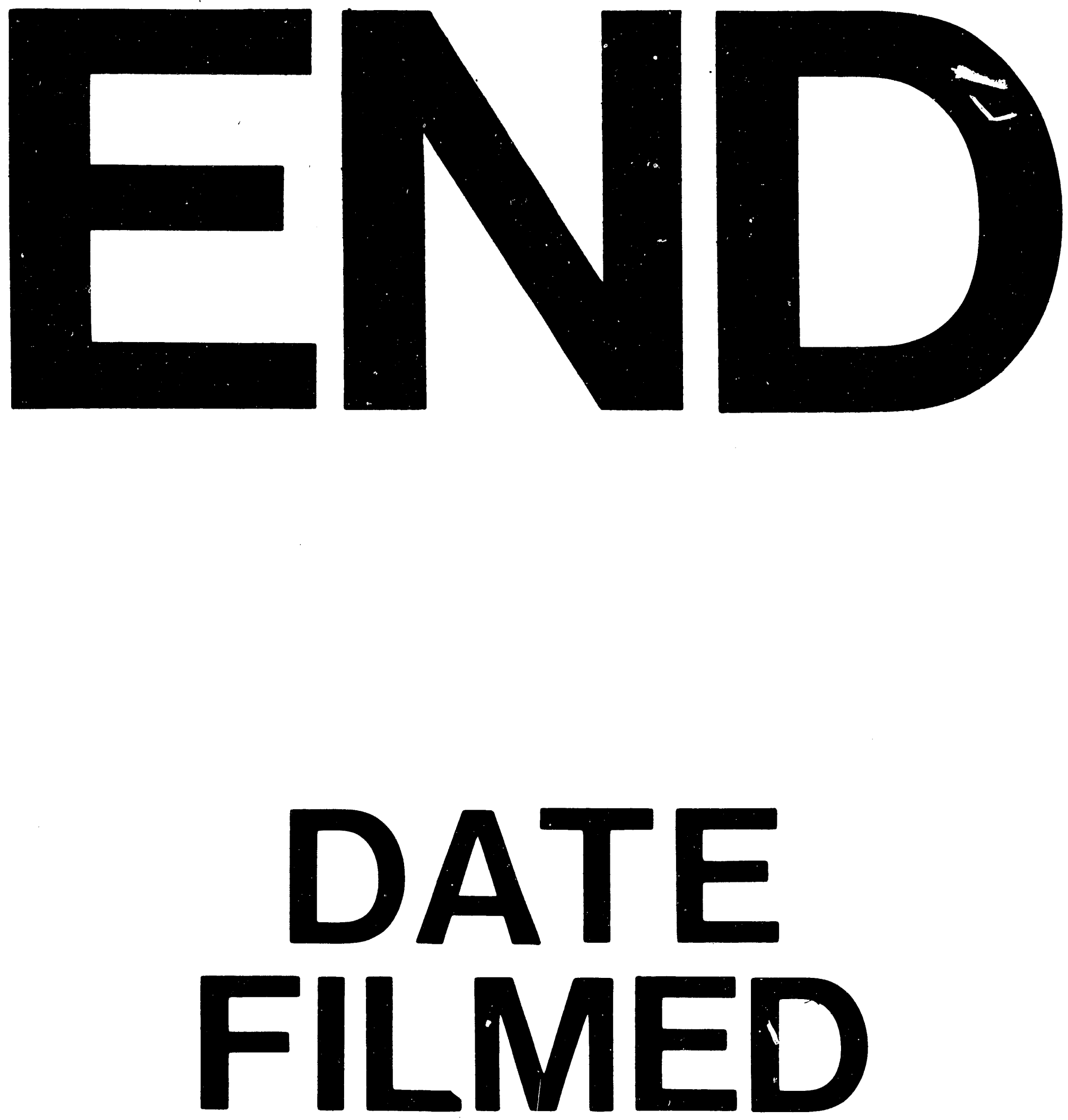

$f$

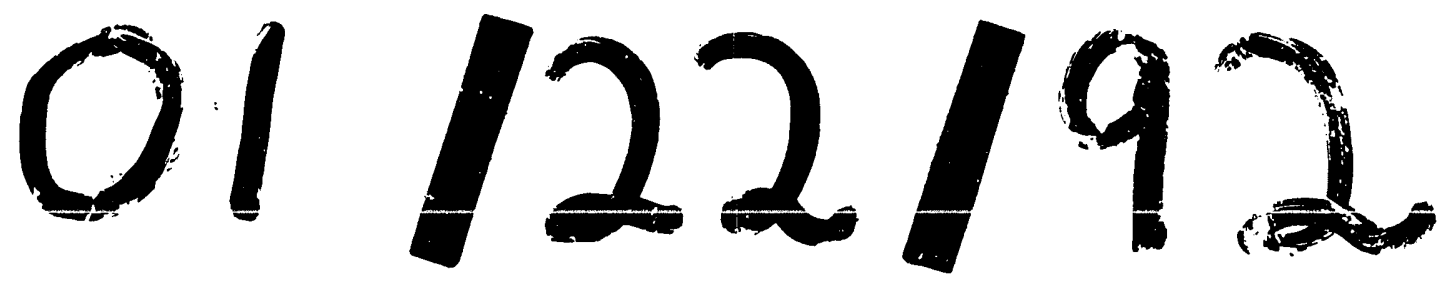


